Rapport - Société canadienne d'histoire de l'Église catholique

\title{
Missionnaires jésuites au Nipigon
}

\section{Lorenzo Cadieux}

Volume 25, 1957-1958

URI : https://id.erudit.org/iderudit/1007445ar

DOI : https://doi.org/10.7202/1007445ar

Aller au sommaire du numéro

Éditeur(s)

La Société canadienne d'histoire de l'Église catholique

ISSN

0318-6148 (imprimé)

1927-7075 (numérique)

Découvrir la revue

Citer cet article

Cadieux, L. (1957). Missionnaires jésuites au Nipigon. Rapport - Société

canadienne d'histoire de l'Église catholique, 25, 91-101.

https://doi.org/10.7202/1007445ar

Tous droits réservés @ La Société canadienne d'histoire de l'Église catholique, 1958
Ce document est protégé par la loi sur le droit d'auteur. L'utilisation des services d'Érudit (y compris la reproduction) est assujettie à sa politique d'utilisation que vous pouvez consulter en ligne.

https://apropos.erudit.org/fr/usagers/politique-dutilisation/ 


\section{Missionnaires jésuites au Nipigon}

L'histoire des missions indiennes au nord du lac Supérieur commence, en 1667, lorsque le Père Claude Allouez visita un embryon de chrétienté au lac Nipigon (Alimibegong). Depuis l'invasion iroquoise, ces Indiens n'avaient pas revu une Robe-Noire. Le missionnaire Jésuite résolut de les réconforter. Il fit le voyage en compagnie de deux guides sauvages et atteignit, après d'effarantes difficultés, l'embouchure de la rivière Nipigon. Il y dit la messe, le jour de la Pentecôte, en présence d'une vingtaine de chrétiens qui pratiquaient ouvertement leur religion ${ }^{1}$. Le Père Allouez était le premier Blanc à s'aventurer dans ces parages.

Un siècle passe. La conquête anglaise bouleverse la carte missionnaire de la Nouvele-France et contraint les apôtres de l'Ouest à l'abandon de leur champ d'apostolat. L'œuvre des trois grands pionniers de la foi, Marquette, Dablon et Allouez ${ }^{2}$, va-t-elle disparaître? Non pas. Dès 1831, un jeune prêtre au zèle de feu, l'abbé Frédéric Baraga, sillonne le littoral du lac Supérieur ${ }^{3}$.

Peu après, les Jésuites reviennent à leurs anciennes missions de la baie Georgienne. En 1844, ils apparaissent sur la première ligne de bataille de l'Ile Manitouline, à Wikwemikong, centre missionnaire considérable, point de départ de prodigieuses aventures spirituelles et point de ralliement de nombreuses tribus indiennes. A la vue de cette région tant désirée, de grosses larmes coulent sur la figure du Père Jean-Pierre Choné. "Il me serait impossible, écrit-il, de dépeindre l'état où je me trouvais à ce moment [...] Un des premiers, j'allais prendre la place de nos anciens Pères et Frères qui ont arrosé de leur sang et sanctifié par leurs souffrances cette terre infidèle ${ }^{4}$...

En 1849, ils reprennent leur mission de Fort-William, fondée un siècle auparavant. C'est de là que partiront les évangélisateurs du lac Nipigon. Durant une soixantaine d'années, ils s'astreignent à un trajet de cent vingt milles, deux fois l'an: l'hiver, en raquettes ou en traîne sauvage; l'été, en canot ou à pied.

C'est au Père Nicolas Frémiot que revient l'honneur d'avoir fondé la mission à Nipigon House. En février 1852, il s'achemine vers ces

1 Relations des Jésuites, 3 vol., Québec, 1855, vol. III, Relation de 1667, 24s. Aussi Francis J. Nelligan, The visit of Father Allouez to Lake Nipigon in 1667, dans The Canadian Catholic Historical Association, Report 1956, 41.

2 L'historien américain Bancroft fait l'éloge de ce fameux triomvirat, voir Georges Bancrooft, History of United States, (London 1853), II: 805s.

3 Lorenzo Cadieux, s.j., et Ernest Comte, s.j., Un Héros du lac Supérieur, Frédéric Baraga, Collection Documents historiques de la Société historique du Nouvel-Ontario, No 27 (Sudbury, 1954).

4 Choné à un Père, Lettres des Nouvelles Missions du Canada, 1843-1849 et 1850-1851, I: 135 . 
régions glaciales. Il a accepté l'offre des courriers de la Compagnie de la Baie d'Hudson qui se rendaient à Nipigon House, poste de traite situé sur la côte ouest du lac. Durant le trajet, les voyageurs souffrent « horriblement du froid ${ }^{5}$ ". Le soir, on dresse la tente, mais il est impossible de fermer l'œil. "Il fallait, raconte le Père Frémiot, tourner le dos au feu pour n'avoir pas les yeux crevés par la boucane, et se retourner bien vite vers le feu pour n'avoir pas les mains gelées. A l'entrée du lac, j'avais les sourcils et les cils tellement chargés de glaçons que je pouvais à peine soulever les paupières et ne voyais plus qu'un jour obscur comme à travers une forêt ${ }^{6}$.»

Après six jours de voyage, il atteint le poste de la Compagnie de la Baie d'Hudson. Il y était vivement attendu par le bourgeois lui-même, M. Louis-Denis de la Ronde, un Canadien français, catholique convaincu qui, à défaut de prêtre, était catéchiste et baptiseur de nouveau-nés, servait de témoin aux nouveaux couples et de consolateur aux moribonds.

Le Père Frémiot se mit aussitôt à l'œuvre. Chaque jour, il se prodigua: messe, catéchismes, leçons de chant et instructions. Il enseigna l'ABC aux enfants avec autant de joie que s'il avait donné des cours à la Sorbonne. Il prêcha les exercices spirituels du Carême et de la Semaine Sainte qui furent couronnés, à la fête de Pâques, d'une première communion solennelle, cérémonie tout à fait inusitée dans cette région. Puis, il érigea une croix de vingt pieds de hauteur auprès du poste. « Elle regarde le lac, écrit-il, et semble inviter de loin les familles nomades qui le sillonnent en tous sens à venir chercher sous son ombre le repos, le salut et la vie. Dès le lendemain, les Indigènes abordèrent en foule; le lac venait enfin de briser en mille morceaux la voûte de glace sous laquelle il s'était abrité depuis sept mois ? .»

D'après les renseignements reçus, le missionnaire s'attendait à rencontrer des anthropophages; au contraire, les Indiens, des Odjibwés (appelés aussi Sauteux, Ojibwa, Chippewa, etc.) étaient pleins de respect et d'affabilité pour la Robe-Noire. Le Père Frémiot convertit le frère d'un redoutable sorcier. Il est au comble de la joie. Ecoutons-le chanter son allégresse:

Quand mon pénible voyage à la raquette au milieu des rigueurs de l'hiver, quand mon séjour de trois mois au Nipigon pour y atteindre les Sauvages, quand toutes les tribulations dont j'y fus assailli n'auraient point d'autre récompense que d'avoir envoyé au ciel l'âme de Joseph l'Iroquois, ne devrais-je pas m'estimer le plus heureux des mortels d'avoir été choisi de Dieu pour être l'instrument d'une si grande faveur de sa Miséricorde ? Oui, c'est dans ces tristes pays, où les élus sont si rares à glaner qu'on estime à sa juste valeur la gloire et le bonheur d'avoir pu contribuer tant soit peu à une si sainte et si sublime entreprise! $O$ anima! tanti vales! Je vois ces grands enfants sillonner nos lacs malgré l'intempérie des saisons et interroger patiemment chacun des rochers qui les bordient et quand leurs yeux avides ont découvert enfin quelques

5 Frémiot à Boulanger, 19 mars 1852, ibid., II: 197.

6 Loc. cit.

7 Le même au même, 2 juillet 1852, Lettres des Nouvelles Missions du Canada, II: 207. 
paillettes de ce brillant métal qui fait palpiter leurs cœurs, une folle ivresse les entraîne, ils s'estiment les plus fortunés des mortels et oublient en un instant cette longue chaîne de soucis et de fatigues qui a comme enlacé leur existence. Et illi quidem ut corruptibilem coronam recipiant, nos autem incorruptam 8 ...

Soutenu par une force surnaturelle, cet apôtre donna deux cents soixante-dix instructions catéchistiques, baptisa dix-huit personnes et lutta énergiquement contre les superstitions dont l'une, le Tsisakiwin, était pratiquée avec passion par les païens du Nipigon. Cette superstition paraît supposer l'intervention directe du démon. On fiche en terre une dizaine de perches liées ensemble par le haut. La nuit venue, le jongleur entre dans sa cabane et commence ses incantations. Aussitôt, sans que le jongleur touche à rien, tout s'ébranle. Les âmes des revenants révèlent, dit-on, des secrets ou prédisent l'avenir ${ }^{9}$.

Le Père Frémiot raconte à son supérieur comment il avait combattu cette pratique diabolique.

Un jeune homme voulut faire la Tchicakkiouine (entendez le Tcisakiwin). Il était, dit-on, expert dans le métier. Déjà il a commencé à faire branler la machine, lorsque je me dirige secrètement derrière la clôture, à une petite distance du théâtre magique. Là, avec une foi vive et une ferme confiance en Dieu, je prononce en latin, à voix basse, diverses formules d'exorcisme et, chose merveilleuse, notre homme a beau continuer ses chants, rien n'ébranle plus. Bientôt, j'entends qu'on se rit de son impuissance [...] A la fin, les assistants, fatigués des inutiles tentatives du jongleur, renversent l'édifice de fond en comble et le jeune homme, ne sachant trop sans doute à quoi attribuer cette mésaventure se hâta de décamper ${ }^{10}$...

Au Nipigon, la superstition était l'obstacle majeur à l'évangélisation. Cependant, il y en avait un autre: la vie nomade. Les familles des chasseurs étaient disséminées dans la forêt environ onze mois par année. Au printemps, elles s'acheminaient vers les postes de traite. Leur bref séjour d'un mois, près des magasins des postes, était le seul temps propice à l'évangélisation.

Lorsque le Père Frémiot fit la relation de son ministère au Nipigon, il proposa un plan de campagne. D'abord, la nécessité pour le missionnaire de rencontrer les Indiens au printemps; ensuite, l'urgence d'ouvrir une mission au lac Seul et une autre au lac Saint-Joseph, où les Indiens, qui parlent la même langue que ceux du Nipigon, n'ont jamais vu la Robe-Noire. Ce projet, il aurait voulu le réaliser, mais le devoir l'appelle ailleurs, au Sault-Sainte-Marie.

Son successeur, le Père Dominique du Ranquet est, lui aussi, un jésuite de France qui peut soutenir la comparaison avec nos plus grands

8 Ibid., 214s. \& Eux, ils travaillent pour une couronne périssable; nous, pour une couronne incorruptible ", I Cor., IX 25.

9 Diamond Jenness, The Ojibwa of Parry Island, Their Social and Religious Life, Ottawa 1935, Canada Dept. of Mines, Bulletin No. 78. La meilleure explication de la tente tremblante et l'interprétation de cette cérémonie se trouve dans un article de Jacques Rousseau, intitulé: Rites païens de la forêt québécoise: la tente tremblante et la suerie, dans les Cahiers des Dix, 19 (1953): 129-148.

10 Frémiot à Boulanger, 2 juillet 1852, op. cit., II: 205. 
missionnaires. Qui dira les aventures hallucinantes dont il fut témoin pendant ses interminables randonnées? Il voyageait tantôt seul, tantôt en groupe quand il suivait les caravanes de canots afin de pouvoir repérer les campements indiens, échelonnés autour du lac tels que Wabinosh, Ombabika, Sand Point, Grand Bay, Nipigon House.

On peut facilement retracer son itinéraire à l'aide de son journal, composé durant ses voyages et rédigé en style télégraphique. Sa plume court en toute liberté sur de grandes feuilles, à la façon de ses chiens sur les plaines enneigées. Ce journal de sept cent cinquante-six pages, le Père du Ranquet l'a écrit, parfois assis sur une roche, souvent à la lueur d'une bougie ou d'une écorce enflammée. Il ressuscite devant notre imagination ébahie la dure vie du missionnaire ontarien, avant que le chemin de fer multiplia les facilités de locomotion.

Dans son journal, il a consigné une foule d'observations sur les mours, les coutumes, les danses indiennes, la chasse au huard, chasse préférée des Indiens. Il note que la glace fondante, au dégel, " se met en chandelles ${ }^{11}$ ", qu'un matin, au lever du soleil, passant en canot devant un énorme rocher, " un hibou blanc, qui ne paraît aucunement effarouché, le suit des yeux tournant doucement la tête ${ }^{12}$...»

Mais la vie de cet apôtre est plus austère que son style, elle semble tenir du miracle. Il travaille prodigieusement et jeûne presque continuellement. Ni pâtisserie, ni pomme de terre. Sa nourriture, quand il en prend, se compose de poisson et de chair de gibier, cuits dans l'eau, sans poivre ni sel. Aurait-il pu garder un tel régime alimentaire sans une grâce particulière ? ${ }^{13}$ S'il se mortifie de la sorte, c'est qu'il croyait, lui, comme Notre-Seigneur, à l'efficacité du jeûne et de la prière pour obtenir la conversion des païens.

Un jour qu'il était en route, vers le Nipigon probablement, on vint l'avertir qu'un Indien se mourait à la suite d'un combat avec un ours qu'il avait tué. Le Père part seul, en canot. Avant d'arriver, son canot chavire à une petite distance du rivage. Il gagne le bord à la nage, en poussant son canot devant lui. Puis, il se lance à l'eau pour repêcher son bagage. Peine perdue. Il revient sur la grève, se repose de cet exercice épuisant, puis il s'enfonce dans la forêt. Après quelques heures de marche, il était auprès du malade qu'il secourut sans rien lui raconter de son aventure. Il repartit sans lui demander de nourriture, car le malade était trop pauvre. Le soir, il parvint à son canot qu'il renversa sur lui et passa ainsi la nuit. Le matin, il s'embarque, très peiné d'avoir perdu sa chapelle et son fusil. Il revient avec lenteur, avironnant de plus en plus faiblement. En abordant au quai, il était pâle comme un mort; il chancela et fut incapable de proférer une parole... Sans le le récit qu'en écrivit M. Finlayson, personne n'en eut jamais rien su.

Ces défaillances, qui se renouvelèrent à plusieurs reprises, montrent assez de quelle énergie il était doué et jusqu'à quelles limites il poussait le dévouement. Un soir, à la veillée, par un grand froid d'hiver, le Père arriva, sa barbe qu'il portait longue est prise en un glaçon et lui ferme

11 Journal du Père du Ranquet, 13 mai 1864; Archives du Collège Sainte-Marie, $3180: 312$.

12 Journal du Père Ranquet, 28 octobre 1874: 654.

13 Lettres des Nouvelles Missions du Canada, I: 98. 
la bouche. Soudain il chancelle et s'affaisse d'épuisement. Le père Choné fut indécis un moment s'il ne devait pas lui donner l'Extrême-Onction. Une autre fois qu'il avait marché plusieurs jours, il arrivait à la mission, quand il rencontre un Canadien qui lui demande de venir donner les secours de la religion à sa femme mourante. Le Père suit son guide pendant un quart de mille, entre dans la maison et s'évanouit par suite d'un jeûne prolongé de plusieurs jours. Le Canadien, n'ayant pu le ramener à la connaissance, fut contraint de le mettre sur une traîne et de le transporter dans cet état à la mission ${ }^{14}$...

Un tel régime, vingt-cinq ans durant, nécessitait des nerfs d'acier, non moins qu'un esprit de sacrifice peu ordinaire. Dans ses voyages, le Père du Ranquet portait sur le dos une chapelle et une couverture, se nourrissait au hasard des occasions, traversait des forêts inextricables, marchait des journées entières, dormait au clair des étoiles.

Il ne s'accordait aucun soulagement: jamais le moindre mouvement pour chasser les moustiques, jamais un signe de douleur, même après une longue expédition, quand le cou et les mains étaient en sang. Il voyageait aux plus mauvaises époques de l'année afin de trouver l'Indien au logis. A la face de notre monde apeuré par la souffrance, épris de confort et de climatisation, cet homme prend la figure d'un héros du moyen âge. Pourtant, il vécut à notre époque; il mourut en 1900.

La succession de ses missions passe, en 1877, entre les mains d'un ex-avocat, le Père Joseph Hébert, un Canadien français. Celui-ci n'avait pas l'habitude de courir les bois. Tout de même, il s'en réjouit d'autant plus que sa santé s'améliore. Il est tout joyeux parce qu'il s'est mis au régime des trappeurs et des coureurs des bois: le gros lard et les excursions en raquettes ou en canot ${ }^{15}$.

Au lac Long, ses prévenances lui gagnent les cœurs. Il partage sa nourriture avec les affamés et son chantier avec les Indiens sans abri, exposés à la pluie et au froid. Il va à la rencontre des canots et salue les arrivants. Ceux-ci sont attirés par la charité et la politesse de la Robe-Noire. Quand le Père leur parle de religion chrétienne et du baptême qui en est la porte d'entrée, les païens réclament cette faveur. Le missionnaire a la joie de conférer le baptême à soixante-dix Indiens dont trente et un adultes ${ }^{16}$.

L'ouvrage n'est pas fini. La superstition empêche plusieurs païens d'entrer dans l'Eglise catholique. Alors son éloquence retrouve les élans passionnés du barreau. Un jour, il persuade l'un d'eux d'abandonner ses pratiques superstitieuses et termine ainsi son plaidoyer: "Fier sauvage, si tu veux devenir chrétien, brûle ce que tu adores.» Et celui-ci de brûler ses rouleaux d'écorce remplis de signes cabalistiques ${ }^{17}$.

Pendant l'hiver 1878, il faillit perdre la vie en traversant la rivière Ogoki; il enfonça deux fois à travers la glace. S'il n'avait pas eu son

14 Théophile Hudon, s.j., Le Père Dominique du Ranquet, s.j., dans le Messager Canadien du Sacré-Caur, octobre 1901: 446.

15 Joseph Hébert, s.j., à son supérieur, Théophile Charaux, s.j., 22 novembre 1877, Cahiers Maynard, III: 693.

16 Le même au même, 2 mai 1879, ibid., 740.

17 Le même à Henri Hudon, s.j., 30 décembre 1880, ibid., 781. 
fusil dans les mains, il aurait coulé à pic; il le tenait devant lui, les deux bouts appuyés sur la glace. Par ce moyen, il réussit à remonter, puis, en marchant avec précaution, il parvint au rivage ${ }^{18}$. Au retour, il passe par Geraldton, (le petit lac Long) où il remarque quelque chose d'extraordinaire: tous les enfants, à l'exception d'un, avaient six doigts aux pieds et aux mains ${ }^{19}$.

Il retourne une dernière fois au Nipigon, en septembre 1880; son successeur l'accompagne, un jeune missionnaire alsacien, le Père Joseph Specht, qui avait été ordonné à Fort-William, quatre mois auparavant. Cette première randonnée est si pénible au Père Specht qu'elle lui rappelle souvent la Passion de Notre-Seigneur. Ecoutons-le raconter ce voyage:

...le 24 septembre (1880), à deux heures de l'après-midi, le Père Hébert et moi, nous avons quitté Red-Rock pour Nipigon en canot. Deux Indiens nous aidaient. Le voyage dura huit jours et offrit des difficultés que seul un missionnaire sait comprendre. Une distance de cent milles et sept portages à travers des sentiers si raboteux qu'ils ne méritent pas même ce nom. Vous comprenez quelle fut notre fatigue. A quelque chose malheur est bon. J'y ai trouvé une méthode pratique pour évoquer, dans mes méditations, la Passion de Notre-Seigneur... Retardés sur le lac Nipigon par les vents contraires, nous arrivâmes le $1^{\text {er }}$ octobre au Fort Nipigon. Nos chrétiens étaient assemblés au débarcadère pour nous souhaiter la bienvenue. Alors distribution de poignées de mains de tous côtés et accueil chaleureux de la part de M. Henri de la Ronde 20, le Bourgeois ou chef du poste de la Compagnie de la Baie d'Hudson. Après la prière du soir, récitée en commun, le Père Hébert me présenta aux Indiens comme son successeur, puis retourna à FortWilliam. Je restai seul. Ah ! cette solitude du missionnaire au milieu des sauvages! Que c'est déprimant au début 21!

Aussitôt après le départ du départ du Père Hébert, eut lieu une cérémonie qui peut sembler puérile à des Blancs, mais à laquelle les Peaux-Rouges attachent une grande importance: l'imposition d'un nom indien au nouveau missionnaire. Les Sauvages n'ont pas le don des langues; ils ne prononcent que difficilement un nom étranger, même s'il n'a qu'une syllabe comme Specht.

On plaça des chaises en rond, en face d'une estrade provisoire réservée aux orateurs de circonstance. Selon la coutume, on apporta des vases remplis de cette eau sucrée nommée Okomwabo ou breuvage du chef. L'étiquette indienne requiert que le chef, lorsqu'il préside une assemblée, se régale de cette eau. Beaucoup de viande, de patates, de farine, de tabac et de pipes: voilà le premier numéro du programme. Ensuite, une petite adresse débitée par deux enfants et les discours en objibwé, en français, en anglais et parfois même en latin ! L'orateur principal s'avance, doit prendre le "catéchumène" par la main, le conduire devant la tribune et proposer le nom qui sera désormais employé

18 Le même au même, 4 mai 1880, ibid., 767.

19 Le même à un supérieur, 24 février 1880, ibid., 759.

20 Son oncle, sir Charles de la Ronde, mourut en 1881, à l'âge de 81 ans. Il était un descendant de la noblesse française. Il employa vaillamment les deux dernières années de sa vie à l'enseignement.

21 Joseph Specht, s.j., à Charaux, 15 octobre 1880, Cahiers Maynard, III: 795s. 
pour désigner le missionnaire. La fête se termine par des cris, des danses, des gestes et des grimaces. Ceci fait, une squaw s'avance vers le Père Specht, lui prit la main et fit un tour de valse au son du tam-tam. Ils l'appelèrent Kapapaminwadjimo: celui qui apporte des nouvelles joyeuses. Toute la bande approuva par trois cris caractéristiques: Wa ! Wa ! Wa!

Au Nipigon, il y a quelque chose qu'on ne trouve nulle part ailleurs. Si l'Ile Bonaventure, en Gaspésie, est le sanctuaire des oiseaux, Nipigon est le paradis des chiens. Quand le Père visita l'école construite à une distance de trois-quarts de mille du fort, il fut salué par deux douzaines de chiens, jappant et aboyant après lui. Cette réception l'énerva quelque peu. Ses mollets tremblèrent. Il se compta chanceux d'échapper à la gueule de ces carnassiers. A son départ, ils firent entendre des hurlements lamentables.

Malgré ces ennuis, ce premier séjour au Nipigon réjouit le Père Specht, car il fut enchanté de la ferveur des Indiens. Le Père portera le lourd fardeau des missions du Nipigon pendant dix-huit ans.

En 1898, la température du lac Nipigon n'a pas changé: un froid de loup, l'hiver; l'été, on voyage en compagnie de multitude d'insectes, dans une atmosphère torride. Les misères de ces excursions, le Père Napoléon Dugas les évoque dans une lettre écrite à son frère:

Nous nous embarquâmes en canot d'écorce, lundi, le 11 juillet à 2 heures du soir, et nous arrivâmes à Nipigon Mission, vendredi à $101 / 2$ heures du soir, après avoir remonté la rivière Nipigon ainsi que le lac Nipigon en compagnie de toutes sortes d'insectes. Monseigneur [0'Connor] avait la figure, les jambes et les bras couverts de sang. On aurait dit qu'il s'était battu à coups ide couteau. Comme j'ai la peau plus épaisse, les moustiques, après avoir sucé tout ce qu'ils pouvaient, ne laissaient d'autres traces sur ma peau que les bosses.

En montant, nous eûmes le plaisir de coucher debout, une fois seulement. Nous avions campé au bas d'un rocher et à peine étions-nous étendus sur notre lit de branches de sapin, qu'une pluie torrentielle nous obligea d'abandonner le lit et de nous tenir debout, pendant deux heures, dans l'eau jusque par-dessus le pied.

Après l'orage, toutes les couvertures étaient en lavettes et nous passâmes le reste de la nuit debout autour d'un feu, assis sur la pierre, la tête appuyée sur nos valises.

Au Nipigon, les Sauvages qui se respectent ont 23 ou 24 chiens. On peut lire à travers leur corps, tant ils sont gras! Ces chiens chantent le jour et la nuit; il n'y a rien de plus intéressant à entendre. Les Sauvages païens joignent leur musique à celle des chiens. Quand nous arrivâmes, ils ont joué du tambour toute la nuit pour chasser les mauvais esprits! Il faut entendre ce tapage de tambour 22 !...

Jusqu'en 1906, les missions du lac Nipigon relevaient de FortWilliam où résidait le missionnaire. A cette date, le Père Dugas fonde une résidence à Nipigon, station ferroviaire située à soixante-dix milles à l'est de Fort-William.

22 Napoléon Dugas, s.j., à Jacques Dugas, s.j., 27 août 1898, dans les Litteræe Annuce Missionis Canadensis S.J., 1897-1898: 71 s., 74. 
Dans ces régions, le froid est la monnaie courante pour convertir les âmes. Le Père Lamarche fait un voyage à Gull-Bay, par une température de $60^{\circ}$ sous zéro. Arrêté par une affreuse tempête, il se creuse un abri dans la neige pour se protéger ${ }^{23}$. Le Père Bélanger connaît une aventure semblable; il croit sa dernière heure venue: dans son trou de neige, les crampes le saisissent, ses pieds refroidissent pendant que la neige s'amoncelle sur sa tête et l'empêche de respirer. N'en pouvant plus, il se relève, cherche son guide enseveli sous la neige et l'éveille. Ils partent, atteignent une île où enfin ils peuvent se faire un bon feu. C'est le salut ${ }^{24}$.

Tout cet héritage de misères et de grandeur missionnaire sera confié, en septembre 1924, au Père Joseph-Marie Couture. Il ira, avec autant de vaillance que ses prédécesseurs, porter aux pauvres Sauvages le message consolateur de son Maître. Il connaîtra, lui aussi, l'ennemi capital: le froid. Avant d'être nommé officiellement aux missions du Nipigon, il en fit l'apprentissage en janvier 1924. Cette expérience fut extrêmement pénible: fièvre, amygdale enflée qui crève; il crache du sang ${ }^{25}$.

Le voici au sud du lac Nipigon, à McDiarmid. Il attend un guide. Cinq jours passent, personne. La température est très mauvaise. Il monte dans le train, passe par Longlac et file vers Ombabika. Les maux de gorge le tourmentent et la fièvre monte. Il se soigne un peu, puis chausse ses raquettes et part vers Wabinosh, un trajet de 40 milles. Il marche 12 milles jusqu'à une île. Là, un guide indien a promis de le rejoindre avec une traîne et de bon gros chiens. Longue attente. Et le vent qui danse, hurle, change de direction, tourne sur tous les points de sa boussole. Le guide arrive enfin! Mais les chiens sont trop petits, trop maigres pour transporter le Père et ses bagages.

Le missionnaire est faible, il a peu mangé depuis cinq jours. Il marche dans la tempète. Après un parcours de 10 milles, il se jette sur la traîne, épuisé. Les chiens ralentissent, puis, refusent d'avancer. Il faut pourtant continuer. Enfin, le Père arrive à Wabinosh; il arrête à la première cabane et là, étourdi, il s'écrase par terre.

Vers deux heures du matin, il se réveille. Une souffrance atroce aux côtés le fait crier. Pendant une heure, il reste adossé au mur; peu à peu, la crampe disparaît. Quelques heures plus tard, le ministère commence: confessions, messe, instruction. L'après-midi, il repart en direction de Nipigon House; encore quinze milles! Il y arrive fiévreux; les dents lui claquent dans la bouche de sorte qu'il ne peut plus parler. On lui offre une "ponce » de Painkiller et le voilà mieux.

23 Litterce Annuce Provincia Canadensis S.J., 1907-1912 : 249s. Le Père Dugas s'y rendit en 1911 par une température de $70^{\circ}$ sous zéro; ibid., 252. Voir aussi W. G. Dean, Human Geography of the Lower Albany River Basin, dans Geographical Bulletin, Department of Mines and Technical Surveys, Ottawa, 10 (1957): 58.

24 Nouvelles de la Province du Bas-Canada, II, 9 (mai 1933): 5.

25 Couture à un Père jésuite, 22 mars 1926. Archives de l'Université de Sudbury. 
Il poursuit sa visite paroissiale: Jack Fish Island, Gull Bay, McDiarmid, une distance de soixante-six milles sur le lac. A mi-chemin, il fait halte dans une île. Il couche dans une tente où un mauvais poêle fume, fume toute la nuit, tellement qu'il se réveille à demi asphyxié. Pour respirer, il est obligé de se mettre le nez dans les branches de sapin qui tapissent le sol. Enfin, après un mois de misères et de souffrances, il revint, exténué, au Nipigon ${ }^{26}$.

L'hiver de 1925 connut aussi des froids à pétrifier n'importe quel être vivant. Cette température polaire n'empêcha pas le Père Couture de porter la joie de sa présence et de sa parole à ses ouailles, qui venaient de le baptiser du nom de Neendamishkang: Celui qu'on aime à voir venir.

Cette fois-ci, le Père était habillé plus chaudement. Il avait hérité d'un vieux paletot dont se servait le Père Joseph-François Richard pour couvrir le radiateur de son auto. Nettoyé, raccommodé, muni d'un capuchon neuf et d'une doublure de laine, ce paletot était léger comme une plume. Il le portait avec plaisir, car il pouvait facilement courir devant ses chiens ou marcher rapidement en raquettes.

Mais ce vêtement était-il trop précieux pour le religieux ? Il le semble bien, puisque, pendant un voyage en train, son sac disparut. Son " riche" butin tomba entre les mains d'un voleur qui croyait subtiliser de belles fourrures 27 !... Les intimes du Père Couture ne se rassasiaient pas de l'entendre raconter cette anecdote savoureuse.

A la mi-avril, il se risque sur le lac Nipigon. C'est la deuxième visite cet hiver. Cette fois-ci, la mort le talonne.

Il était un peu tard pour s'y risquer, écrit-il. J'ai fait encore 140 à 150 milles avec de bons Sauvages et de bons chiens. Je ne pouvais revenir sur mes pas, car, à mesure que j'avançais vers le nord, la glace devenant mauvaise se détériorait derrière moi! Cependant, je suis arrivé sain et sauf au nord du lac, au chemin de fer du National Canadien 28 ...

De 1924 à 1940, le Père Couture se donne corps et âme à ses ouailles. Rien n'échappe à sa vigilance. Le problème scolaire l'inquiète, car les écoles du Nipigon sont dans un état pitoyable. Il s'en ouvre à l'agent des Affaires Indiennes, J. G. Burk, de qui relèvent les bandes du Nipigon et de Longlac. Les solutions que propose le missionnaire sont adoptées. "Des écoles neuves, il en faut au Nipigon, sinon impossible d'instruire les enfants; d'ailleurs, les professeurs qualifiés refusent d'enseigner dans ces cabanes, des glacières en hiver ${ }^{29}$."

26 Durant l'hiver de 1931, le Père Couture fit une tournée de 550 milles pour visiter quatre missions. a Pendant ce voyage, écrit-il, j'ai dû marcher plus de 400 milles en raquettes." Au retour, deux de ses quatre chiens-loups sont morts d'épuisement. Le missionnaire, lui, garda le lit quelques jours. Voir lettre du Père Couture au Père Wlodimir Ledochowski, 7 novembre 1932. Couture à sa mère, 20 février 1925.

28 Le même à la même, 19 mai 1925.

29 J. G. Burk répète les paroles du Père Couture dans une lettre adressée au missionnaire, 26 novembre 1927. 
Cet agent des Affaires indiennes estimait le Père Couture; il le consultait sur différentes questions et s'adressait à lui pour régler toute sorte de problèmes, par exemple pour jauger la valeur d'un professeur ${ }^{30}$, pour distribuer des vêtements aux pauvres ${ }^{31}$, pour hâter la construction de nouvelles maisons ${ }^{32}$.

Lorsque le Père Couture prit charge des missions du Nipigon, la population était chrétienne dans une proportion de $92 \%$; sept ans plus tard, elle était toute chrétienne.

Le territoire de ses missions ne se bornait pas au lac Nipigon, il englobait la région de Longlac et les missions de la rivière Albany: le lac Saint-Joseph̆, Fort Hope et Ogoki. Pendant treize ans, de 1920 à 1933, chaque été, le Père Couture parcourait environ 2,000 milles en canot ou à pied; l'hiver, environ 1500 milles en traîne sauvage ou en raquettes; puis, de 1933 à 1940, il se servit de l'avion pour rejoindre ses " paroissiens ", disséminés entre la baie James et les frontières du Manitoba ${ }^{33}$. Sans l'avion, il aurait été obligé de discontinuer son apostolat auprès des Indiens, car une arthrite crucifiante l'empêchait de marcher longtemps.

Homme pratique, le Père Couture employa les moyens les plus modernes que pouvaient lui procurer les progrès de la science. Il acheta un clavigraphe portant des caractères syllabiques et une machine à polycopier avec laquelle il imprimait des circulaires, des exhortations, de courtes vies de saints. Il fit enregistrer sur disques les principales prières, des couplets de cantiques et deux messes chantées. Il se procura même une lanterne magique. Quelle joie dans une mission quand le Père Couture annonçait sa venue. C'était alors tout un émoi dans la bourgade. Personne n'aurait voulu manquer le cinéma! A la nuit tombante, le Père installait sa lanterne et montrait plusieurs séries de diapositives représentant des tranches d'Histoire de l'Eglise, des scènes de la Passion de Notre-Seigneur, des illustrations catéchistiques. Il ajoutait un ou deux documentaires sur des questions d'hygiène et d'astronomie. Il fallait donner aux Sauvages quelques bribes d'astronomie car ils avaient peur des éclipses de soleil.

Le Père Couture, semble-t-il, est la figure dominante de toute cette épopée missionnaire. Sans vouloir en aucune façon sous-estimer l'œuvre des autres missionnaires, tels que les Frémiot, les du Ranquet, plusieurs contemporains pensent que le Père Couture, vu l'intensité de ses travaux et de son admirable charité chrétienne, se place au premier rang de cette phalange d'apôtres. Lorsque la maladie l'obligea à se confiner dans la région de Longlac, en 1941, on eut besoin de cinq hommes pour le remplacer: deux à la rivière Albany, les Pères oblats Maurice Ouimet et Robert Charland; deux au lac Nipigon, les Pères jésuites, Michaël

30 Le même au même, 12 octobre 1928.

31 Le même au même, 25 mars 1930.

32 Le même au même, 5 septembre 1930.

33 Le Père Couture obtint son permis de pilote en janvier 1936. Il était le premier prêtre-aviateur canadien. Voir Travers à Cadieux, 26 septembre 1956, Archives de l'Université de Sudbury. 
Hawkins, à Armstrong, et Alexander Rolland, à MacDiarmid; il partagea aussi avec un prêtre séculier une partie de ses missions situées le long du chemin de fer.

Il n'y a pas à s'étonner si le bilan de sa carrière missionnaire est imposant: plus de trois cents conversions et près de mille baptêmes ${ }^{34}$. Cette conquête spirituelle, il faut l'attribuer à sa merveilleuse charité qui brillait dans ses yeux, enflammait les cœurs et attirait dans sa masure, la plus misérable de la réserve de Longlac, évêques, prêtres, laïcs, Indiens, catholiques, protestants, etc. On aimait la compagnie de ce bon géant, de ce coureur de bois, de ce champion de l'aviron, de cet aviateur audacieux qui, par devoir ou par charité, avait frôlé la mort dans les tempêtes ou dans les pannes d'avion.

Le Père Couture est mort en 1949, au milieu de ses Indiens de Longlac. Tant que le corps du cher défunt fut exposé dans l'église, les Indiens, sans que personne ne les eut organisés, se sont tenus en relève, jour et nuit, auprès du cercueil. A un Père qui leur demandait pourquoi ils aimaient tant à rester auprès de leur missionnaire: "Le Père Couture, répondirent-ils, surpris de la question, aimait les Indiens.» C'était vrai: il avait donné sa vie pour eux.

Rien n'est plus saisissant, certains dimanches, que de voir les Indiens descendre dans la crypte de l'église de Longlac et, comme dans une chapelle ardente, défiler devant le grand mort. Non, vraiment, il n'est pas éteint le feu de la charité qu'avait allumé dans les cours l'apôtre des Odjibwés; et le souvenir de sa bonté d'âme vit toujours. La pierre tombale a recouvert son corps d'un voile de silence, non d'oubli. La grande famille indienne, qu'avait transformée la grâce de Dieu, reste sous le charme de Celui qu'elle aimait à voir venir...

Lorenzo Cadieux, s.j.,

Université de Sudbury. 